EPJ Web of Conferences 60, 12015 (2013)

DOI: $10.1051 /$ epjconf $/ 20136012015$

(C) Owned by the authors, published by EDP Sciences, 2013

\title{
Beyond the Standard Model Higgs Physics using the ATLAS Experiment
}

\author{
Matthew Beckingham ${ }^{1,2, a}$ \\ ${ }^{1}$ University of Washington, Seattle WA, United States of America \\ ${ }^{2}$ On behalf of the ATLAS Collaboration.
}

\begin{abstract}
With the discovery of a Higgs boson that has properties consistent with the Standard Model at the LHC, searches for additional Higgs bosons due to beyond the Standard Model physics, along with potential property measurements not consistent with the Standard Model, become more interesting and relevant. This article summarises the current searches for such new Higgs bosons performed with the ATLAS detector, using proton-proton collisions at centre of mass energies of 7 and $8 \mathrm{TeV}$ at LHC. No significant deviations from the predictions of the Standard Model are observed in any search channel and hence limits on physics beyond the Standard Model are calculated.
\end{abstract}

\section{Introduction}

The recent discovery of a Higgs boson with a mass of approximately $125 \mathrm{GeV}$, and hence the mechanism responsible for electroweak symmetry breaking, represents a triumph of the physics program at the LHC $[1,2]$. Current measurements on the properties of the discovered boson, such as its couplings, show consistency with those for a Higgs boson in the Standard Model (SM) [3]. However, further measurements and data are needed to show this conclusively. Even with such a SM-consistent Higgs boson, there are still issues within the SM - one example is the quantum corrections to the Higgs boson mass that have quadratic divergences. Possible extensions to the SM Higgs sector include adding a further Higgs doublet, collectively known as Two-Higgs-Doublet Models (2HDMs) [4]. A specific case of which, the Minimal Supersymmetric SM (MSSM) [5, 6], can solve these divergences.

Within the 2HDMs an extra complex Higgs doublet is added to the Higgs sector of the SM - this leads to a total of five Higgs bosons: two CP-even bosons, $h$ and $H$, one CP-odd pseudo-scalar $A$ and two charged bosons $H^{ \pm}$. For 2HDMs without the effects of flavour-changing neutral currents, four different types may be defined that depend on the couplings of the two doublets. Of interest to the measurements shown here are the type-I models, where all quarks couple to just one of the doublets, and type-II models, where right- handed up-type quarks couple to one of the Higgs doublets and right-handed down-type quarks couple to the other doublet. Additional parameters used to characterise the 2HDMs are the ratio of the doublet vacuum expectation values, $\tan \beta$, the CP-even Higgs mixing angle, $\cos \alpha$, and the mass $m_{H}$. A specific case of 2HDMs is within the MSSM, which is a constrained type-II 2HDM at tree level. In this case the theory may be described by

\footnotetext{
a e-mail: matthew.beckingham@cern.ch
}

two free parameters at tree level - these are usually chosen to be either the mass $m_{A}$ or $m_{H^{ \pm}}$and $\tan \beta$, where in this instance $\tan \beta$ is the ratio of the vacuum expectation values of the doublet that couples to up-type fermions to that that couples to down- type fermions. Both the general 2HDM models and the MSSM are compatible with the discovery of a Higgs boson with a mass of $125 \mathrm{GeV}$ with SM-like properties [7, 8].

The analyses presented in this paper summarise the recent ATLAS searches for additional Higgs bosons in such beyond the SM models. Firstly a 2HDM inspired search in the channel $H \rightarrow W W$ is presented. Searches for neutral and charged Higgs bosons in the MSSM through the channels $H \rightarrow \tau \tau / \mu \mu, H^{ \pm} \rightarrow \tau \nu$ and $H^{ \pm} \rightarrow c \bar{s}$ are then described. Finally, a direct search for Higgs boson decays to invisible particles is presented.

\section{Two Higgs Doublet Model $h / H \rightarrow W W^{(*)}$}

A search for additional Higgs bosons due to a $2 \mathrm{HDM}$ model in the decay mode $h / H \rightarrow W W^{(*)} \rightarrow e v_{e} \mu v_{\mu}$, using an integrated luminosity of $13.0 \mathrm{fb}^{-1}$ of proton-proton collisions at a centre-of-mass energy of $8 \mathrm{TeV}$, is presented [9]. Here the possibility is investigated that the Higgs boson observed at $m=125 \mathrm{GeV}$ is the low mass Higgs, $h$, of a 2 HDM.

The search begins by following the preselection used in the $\mathrm{SM} H \rightarrow W W^{(*)}$ analysis [10]. Events are triggered by either an electron or muon with a $p_{T}>24 \mathrm{GeV}$. Further, events are selected with exactly one isolated electron and one isolated muon with opposite charges. The leading of these two leptons is required to have a $p_{T}>25 \mathrm{GeV}$ while the other should have $p_{T}>15 \mathrm{GeV}$. Finally, it is required that the invariant mass between the two leptons satisfies $m\left(l_{1}, l_{2}\right)>10 \mathrm{GeV}$ and $E_{T, r e l}^{\text {miss }}>25 \mathrm{GeV}$. Here 


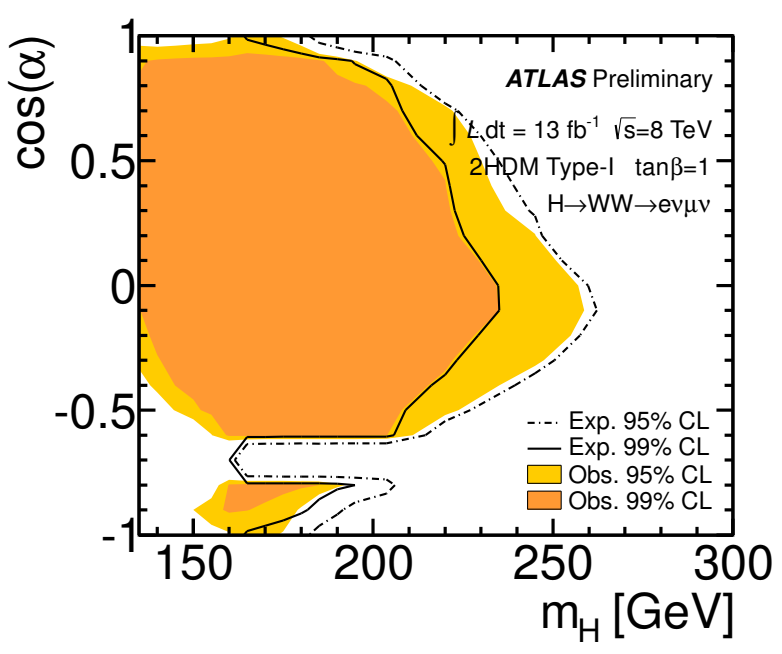

Figure 1. Exclusion contours from the $H \rightarrow W W$ search in the $\cos \alpha-m_{H}$ plane for Type-I Two Higgs Doublet models with $\tan \beta=1$ [9].

$E_{T, r e l}^{\text {miss }}$ is defined as

$$
E_{T, r e l}^{\text {miss }}= \begin{cases}E_{T}^{\text {miss }} \sin \left(\Delta \phi_{\text {min }}\right), & \text { if } \Delta \phi_{\min }<\pi / 2 \\ E_{T}^{\text {miss }} & \text { if } \Delta \phi_{\text {min }}>\pi / 2\end{cases}
$$

where $\Delta \phi_{\min }$ is the minimum difference in azimuthal angle, $\phi$, between a lepton or a jet with $p_{T}>25 \mathrm{GeV}$ and the missing transverse momentum, $E_{T}^{\text {miss }}$, vector. After the preselection, the selected events are split into two channels based on the jet multiplicity. For the 0- jet channel, exactly zero jets are required with the additional requirements that the azimuthal angle between the two leptons is $\left|\Delta \phi\left(l_{1}, l_{2}\right)\right|<2.4$ and the invariant mass of the dilepton system $m\left(l_{1}, l_{2}\right)<75 \mathrm{GeV}$. The 2 -jet channel is defined by requiring exactly two jets which are in opposite hemispheres of the detector, $\eta\left(j_{1}\right) \times \eta\left(j_{2}\right)<0$. In addition, it is required that $m\left(l_{1}, l_{2}\right)<80 \mathrm{GeV}$ and the transverse mass of the dilepton- $E_{T}^{\text {miss }}$ system $m_{T}<180 \mathrm{GeV}$.

To further separate signal and background events, neural networks (NN) are used. Three NNs are trained, at signal mass points of $m_{H}=150,180$ and $240 \mathrm{GeV}$, for each of the 0 -jet and 2-jets channels. In the 0 -jet (2-jet) channel six (nine) kinematic variables are used as inputs to the NNs. Good modelling of the important diboson and $t \bar{t}$ backgrounds are found in control regions enriched for these backgrounds.

No significant excess of events is seen compared to the SM background prediction in the mass range $135<m_{H}<300 \mathrm{GeV}$. Exclusion limits at the $95 \%$ and 99\% confidence level (CL) are hence set in the $\cos \alpha-m_{H}$ plane, for values of $\tan \beta$ ranging from one to 50 for both type-I and type-II models. A large part of the 2HDM parameter space is excluded by this first direct search for a heavy neutral CP-even state in the 2HDM. A example of the exclusion limit at $\tan \beta=1$ is shown in Figure 1. A large part of the $\cos \alpha-m_{H}$ plane can be excluded in the

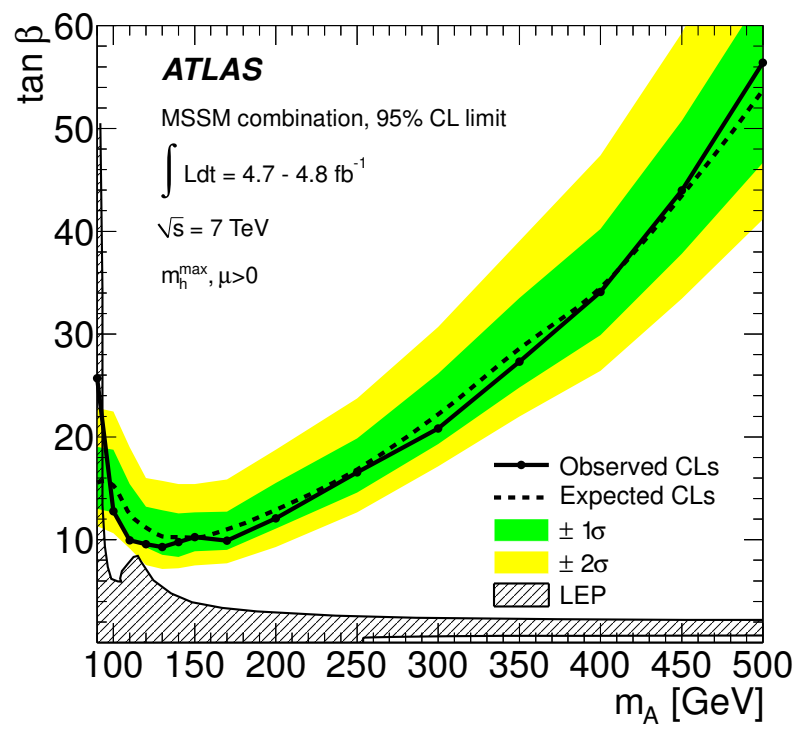

Figure 2. Expected (dashed line) and observed (solid line) $95 \%$ CL limits on $\tan \beta$ as a function of $m_{A}$ for the $m_{h}^{\max }$ scenario of the MSSM [11].

mass range $135<m_{H}<200 \mathrm{GeV}$ for type-I models. In addition, for models with $\cos \alpha \sim 0$ and $\tan \beta=1$, masses up to $m_{H}=250 \mathrm{GeV}$ are excluded.

\section{$3 H \rightarrow \tau \tau / \mu \mu$}

A search for Higgs bosons within the MSSM through the decay to pairs of tau leptons or muons is presented [11]. The tau decay final states are split up into three channels, depending on whether each tau decays hadronically $\left(\tau_{\text {had }}\right)$ or leptonically $\left(\tau_{l e p}\right)$, giving the leptonlepton $\left(\tau_{\text {lep }} \tau_{\text {lep }}\right)$, lepton-hadron $\left(\tau_{\text {lep }} \tau_{\text {had }}\right)$ and hadronhadron $\left(\tau_{\text {had }} \tau_{\text {had }}\right)$ channels. Furthermore, all channels are also split depending on the presence $(b$ - tag) or absence $(b$ veto) of an identified $b$-jet in the final state. An integrated luminosity of $4.6-4.7 \mathrm{fb}^{-1}$ of proton-proton collisions at a centre-of-mass energy of $7 \mathrm{TeV}$ is used.

In the $\mu^{+} \mu^{-}$channel, $h / A / H \rightarrow \mu^{+} \mu^{-}$, a pair of oppositely charged, isolated muons with transverse momenta $p_{T}(\mu)>20,15 \mathrm{GeV}$ are selected. The muon pair is required to have an invariant mass of $m\left(\mu_{1}, \mu_{2}\right)>70 \mathrm{GeV}$ and in addition, the requirement $E_{T}^{\text {miss }}<40 \mathrm{GeV}$ is applied. To reduce the large $Z / \gamma^{*}$ background, the $b$-tag channel is defined by requiring at least one identified $b$-jet. Events failing this requirement enter the $b$-veto channel. In both channels a parametrised fit of the signal and background is performed to the $m\left(\mu_{1}, \mu_{2}\right)$ distribution, by scanning over the mass distribution and fitting the background in side- band regions. The background is parametrised as simplified pure $\gamma^{*}$ and $Z$ propagators with a $\gamma^{*} / Z$ interference term and is convoluted with Gaussian term. The signal is parametrised as a narrow peak with an extrapolation between mass points. The observed numbers of events are consistent with the expected yield from the SM backgrounds within uncertainties. 
The $\tau_{l e p} \tau_{l e p}$ channel is selected by requiring one isolated electron and muon with opposite signs and transverse momenta of $p_{T}(e)>10 \mathrm{GeV}$ and $p_{T}(\mu)>15 \mathrm{GeV}$, respectively. The selected events are then split exclusively into $b$-tag and $b$-veto channels. The scalar sum of the lepton transverse momenta and the $E_{T}^{\text {miss }}$ is required to be $p_{T}(e)+p_{T}(\mu)+E_{T}^{\text {miss }}>125(150) \mathrm{GeV}$ in the $b$-tag ( $b$-veto) channel. The difference in azimuthal angle between the two leptons is required to be $\Delta \phi_{e \mu}>2.0(1.6)$ in the $b$-tag ( $b$-veto) channel. The combination of the transverse opening angles of the directions of the leptons and the direction of the $E_{T}^{\text {miss }}$ should satisfy $\sum_{l=e, \mu} \cos \Delta \phi_{l, E_{T}^{\text {miss }}}>-0.2(-0.4)$ in the $b$-tag ( $b$-veto) channel. Finally, due to the higher $t \bar{t}$ background, the extra restriction on the scalar sum of all jets in the event $H_{T}<100 \mathrm{GeV}$ is used for the $b$-tag channel.

The $\tau_{\text {lep }} \tau_{\text {had }}$ channel is defined by requiring a final state containing one $\tau_{\text {had }}$ with $p_{T}>20 \mathrm{GeV}$ in addition to an opposite sign electron (muon) with $p_{T}>25(20) \mathrm{GeV}$. The transverse mass between the lepton and the $E_{T}^{\text {miss }}$ is required to satisfy $m_{T}\left(l, E_{T}^{\text {miss }}\right)<30 \mathrm{GeV}$ to reduce the $W$ and $t \bar{t}$ backgrounds. Events then form the $b$-tag channel if the leading jet is identified as a $b$-jet and has a transverse momentum in the range $20 \mathrm{GeV}$ to $50 \mathrm{GeV}$. Events are placed into the $b$-veto channel if the leading jet is not identified as a $b$-jet and if $E_{T}^{\text {miss }}>20 \mathrm{GeV}$.

Finally, the $\tau_{\text {had }} \tau_{\text {had }}$ channel is selected by requiring two opposite sign $\tau_{\text {had }}$ with transverse momenta $p_{T}>45,35 \mathrm{GeV}$, respectively. Events which contain electrons or muons in addition are vetoed and the requirement $E_{T}^{\text {miss }}>25 \mathrm{GeV}$ is applied. The selected events enter the $b$ tag channel if the leading jet is identified as a $b$-jet and has a transverse momentum in the range $20<p_{T}<50 \mathrm{GeV}$. Events without jets or where the leading jet is not identified as a $b$-jet are included in the $b$-veto channel. Due to the higher backgrounds in the $b$-veto channel, the selection on the leading $\tau_{\text {had }}$ is tightened to $p_{T}>60 \mathrm{GeV}$.

In the $\tau_{\text {lep }} \tau_{\text {lep }}$ and $\tau_{\text {lep }} \tau_{\text {had }}$ channels of the $h / A / H \rightarrow \tau^{+} \tau^{-}$analysis, the "Embedding" technique is used to model the important $Z / \gamma^{*} \rightarrow \tau^{+} \tau^{-}$background. Here, $Z / \gamma^{*} \rightarrow \mu^{+} \mu^{-}$events are selected in data with a high purity and both muons replaced with simulated taus with the same kinematics. Hence a signal free estimate of this background can be made. The Embedding method is used to validate the Monte Carlo (MC) background estimate in the $\tau_{\text {lep }} \tau_{\text {had }}$ channel. Backgrounds from multijets are estimated using dedicated two dimensional sideband control regions. In both the $\tau_{\text {lep }} \tau_{\text {lep }}$ and $\tau_{\text {lep }} \tau_{\text {had }}$ channels, the $t \bar{t}$ background is estimated using MC normalised to data in dedicated control regions. Finally, the background from $W+$ jets is estimated using MC samples, normalised to data in regions of high $m_{T}\left(e, E_{T}^{\text {miss }}\right)$ and $m_{T}\left(\mu, E_{T}^{\text {miss }}\right)$.

The invariant mass of the $\tau \tau$ system is reconstructed using a technique known as the Missing Mass Calculator in all three final states. This algorithm uses the assumption that any $E_{T}^{\text {miss }}$ in an event comes from the neutrinos from the tau decays and performs a scan over the angles between the neutrinos and the visible tau decay products to chose the most likely invariant mass. The $b$-tag and $b$ -



Figure 3. The $95 \%$ CL upper limit on the branching ratio $B\left(t \rightarrow b H^{+}\right)$as a function of charged Higgs boson mass, derived from a combination of the transverse mass distribution in $\tau_{\text {had }}+$ jets events and the event yield ratio $R_{e+\mu}$ [12].

veto channels from all three $\tau \tau$ final states and the $\mu^{+} \mu^{-}$ final state are statistically combined and no significant excess above the background-only expectation is seen. The 95\% CL limits set on $\tan \beta$ as a function of $m_{A}$ in the $m_{h}^{\max }$ scenario of the MSSM are shown in Figure 2. Additionally, 95\% CL limits are set in the general case of a single scalar boson being produced in either the gluon-fusion or $b$ - associated production modes with a subsequent decay to $\mu^{+} \mu^{-}$or $\tau^{+} \tau^{-}$pairs.

\section{$4 H^{ \pm} \rightarrow \tau v$}

A search for charged Higgs bosons is performed through measuring the lepton universality of leptonically decaying $t \bar{t}$ events, using an integrated luminosity of $4.6 \mathrm{fb}^{-1}$ of data taken in proton- proton collisions at $\sqrt{s}=7 \mathrm{GeV}$ [12]. In the decay of a top quark, the $W$ boson will decay equally to all three generations of leptons. However, if a top quark decays via a charged Higgs boson the subsequent decay to $\tau$ leptons dominates. Thus this measurement searches for an excess of $t \bar{t}$ events with at least one $\tau_{\text {had }}$ in the final state with respect to the rate with only electrons and/or muons in the final state.

Events are selected by requiring an electron or muon in the final state with $E_{T}>25 \mathrm{GeV}$ or $p_{T}>20 \mathrm{GeV}$, respectively, and at least two jets, exactly two of which should be identified as $b$-jets. In addition, the presence of either exactly one $\tau_{\text {had }}$ with $p_{T}>25 \mathrm{GeV}$ or a further lepton with a different flavour to the first lepton and $p_{T}\left(E_{T}\right)>25 \mathrm{GeV}$ is required. Finally a large $E_{T}^{\text {miss }}$ is required.

The background due to misidentified electrons and muons is estimated using a data-driven method based on a control region with looser lepton identification than the signal region. Efficiencies for identifying a real or fake leptons using the tighter identification, based on $Z \rightarrow l l$ and multijet measurements in data, are then applied to this control region to estimate the background contribution in the signal region. The background due to electrons misidentified as $\tau_{\text {had }}$ is estimated by measuring the rate of 


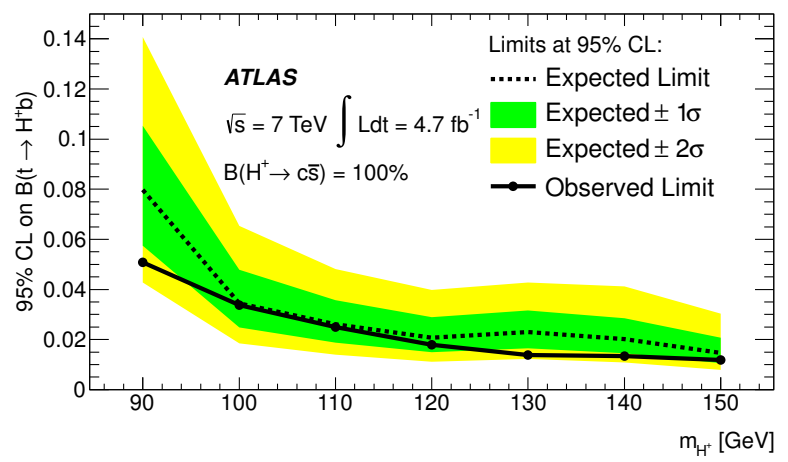

Figure 4. The $95 \% \mathrm{CL}$ upper limit on the branching ratio $B\left(H^{+} \rightarrow c \bar{s}\right)$ as a function of charged Higgs boson mass [14].

such fakes in a $Z \rightarrow$ ee sample in data and applying this fake rate to $\mathrm{MC}$ samples. The background from jets to $\tau_{\text {had }}$ fakes can arise from light and heavy-flavour quarks or gluons. To minimise the fake $\tau_{\text {had }}$ candidates from heavy flavour quarks and from gluons, the difference between events where the $\tau_{\text {had }}$ and the lepton have the opposite sign (OS) and those where they have the same sign (SS) is considered, rather than the raw event yields. To estimate the remaining light quark to $\tau_{\text {had }}$ background, the fake rate is measured in a data sample rich in $W+2$ jet events and is used to scale the Monte Carlo events in the signal region.

For the four final states considered, $e+\tau_{\text {had }}, e+\mu$, $\mu+\tau_{\text {had }}$ and $\mu+e$, the OS- SS yield is measured and the ratios

$$
R_{e}=\frac{N\left(e+\tau_{\text {had }}\right)}{N(e+\mu)} \text { and } R_{\mu}=\frac{N\left(\mu+\tau_{\text {had }}\right)}{N(\mu+e)}
$$

are found to agree within uncertainties to the values predicted by the SM. Exclusion limits on the branching ratio $B\left(t \rightarrow b H^{+}\right)$are then set using either $R_{e}, R_{\mu}$ or the global event yield

$$
R_{e+\mu}=\frac{N\left(e+\tau_{\text {had }}\right)+N\left(\mu+\tau_{\text {had }}\right)}{N(e+\mu)+N_{O R}(\mu+e)} .
$$

Here $N_{O R}(\mu+e)$ represents the number of events in the $\mu+e$ channel with the events removed that fire both the single lepton and single-muon triggers and hence enter the $e+\mu$ channel. The $95 \% \mathrm{CL}$ upper limit on the branching ratio $B\left(t \rightarrow b H^{+}\right)$is found to vary from $3.2 \%$ at $m_{H}=90 \mathrm{GeV}$ to $4.4 \%$ at $m_{H}=140 \mathrm{GeV}$. Finally, to increase the reach of the exclusion limit, a combined limit is set using $R_{e+\mu}$ and the orthogonal $\tau_{\text {had }}+$ jets channel of the previous ATLAS search for charged Higgs bosons [13]. Figure 3 shows the combined 95\% CL upper limit on $B\left(t \rightarrow b H^{+}\right)$, which is seen to vary from $0.8 \%$ at $m_{H}=90 \mathrm{GeV}$ to $3.4 \% m_{H}=160 \mathrm{GeV}$.

\section{$5 H^{ \pm} \rightarrow c \bar{S}$}

The ATLAS search for a charged Higgs boson decaying via the channel $H^{ \pm} \rightarrow c \bar{s}$ is performed using semileptonically decaying $t \bar{t}$ events in $4.7 \mathrm{fb}^{-1}$ of data collected



Figure 5. The confidence level as a function of $B(H \rightarrow$ invisible $)$ for the SM Higgs boson with $125 \mathrm{GeV}$ mass. The dashed line shows the expected values and the solid line the observed values. The red solid lines indicate the $68 \%$ and $95 \%$ CL [15].

at a proton-proton centre of mass energy of $\sqrt{s}=7 \mathrm{TeV}$ [14]. Events are selected to search for one top quark decaying via $t \rightarrow H^{+} b$, with the subsequent decay to two jets $H^{+} \rightarrow c \bar{s}$. The other top quark decays to a $W b$ pair, followed by the decay of the $W$ to a lepton $(e / \mu)$ and a neutrino. Hence the signal process has the same topology as SM $t \bar{t}$ events, but will lead to a dijet mass of the $c \bar{s}$ system peaking at $m_{H^{+}}$.

Events are selected that contain exactly one electron with $E_{T}>25 \mathrm{GeV}$ or one muon with $p_{T}>20 \mathrm{GeV}$, at least four jets with $p_{T}>25 \mathrm{GeV}$ and with at least two jets identified as $b$-jets. To reduce the multijet background, it is required that $E_{T}^{\text {miss }}>20(30) \mathrm{GeV}$ in the muon (electron) channel. This background is further reduced by requiring that the transverse mass of the lepton and $E_{T}^{\text {miss }}$ is in the range $m_{T}\left(l, E_{T}^{\text {miss }}\right)>30 \mathrm{GeV}$ in the electron channel and the $\operatorname{sum} E_{T}^{\text {miss }}+m_{T}\left(l, E_{T}^{\text {miss }}\right)>60 \mathrm{GeV}$ in the muon channel. A kinematic fit is performed on the selected events to reconstruct and constrain the masses of the dijets from $W / H^{+}$candidates by fully reconstructing the $t \bar{t}$ system. The invariant mass of the lepton- $E_{T}^{\text {miss }}$ system is constrained to the $W$ mass and in addition the invariant mass of the blv and $b j j$ systems are constrained to the top quark mass.

Since the data are found to be in good agreement with the predictions of the SM, the dijet invariant mass, $m(j j)$ is used as a discriminating variable to set generic limits on the branching ratio $B\left(t \rightarrow H^{+} b\right)$. Here the assumption is made of $B\left(H^{+} \rightarrow c \bar{s}\right)=100 \%$. Figure 4 shows the 95\% CL upper limit on $B\left(t \rightarrow H^{+} b\right)$ ranging from $5 \%$ at $m_{H^{+}}=90 \mathrm{GeV}$ to $1 \%$ at $m_{H^{+}}=150 \mathrm{GeV}$, which currently represent the most stringent limits on this branching ratio.

\section{Invisible Higgs Decays}

A direct search for the decay of a narrow scalar boson, produced in association with a $Z$ boson, to invisible particles is presented [15]. The search is performed in the decay channel $\mathrm{ZH} \rightarrow l l+$ invisible and is based on an integrated 
luminosity of $4.7 \mathrm{fb}^{-1}$ of data at $\sqrt{s}=7 \mathrm{TeV}$ and a further $13 \mathrm{fb}^{-1}$ of data at $\sqrt{s}=8 \mathrm{TeV}$.

Events are selected that contain either a pair of oppositely charged electrons or muons, with each having a transverse momentum $p_{T}>20 \mathrm{GeV}$. To reduce the $W Z$ background, events containing further leptons are vetoed. To ensure that the lepton pairs are consistent with arising from a $Z$ boson decay, it is required that the invariant mass of the dilepton system is in the range $76<m_{l l}<106 \mathrm{GeV}$. To reject the $Z$ background, a cut of $E_{T}^{\text {miss }}>90 \mathrm{GeV}$ is applied. The difference in azimuthal angle between the $E_{T}^{\text {miss }}$ and $p_{T}^{\text {miss }}$ vectors, where $p_{T}^{\text {miss }}$ is defined as the track-based missing transverse momentum computed using all inner detector tracks with $p_{T}>500 \mathrm{MeV}$, is required to be $\Delta \phi\left(E_{T}^{\text {miss }}, p_{T}^{\text {miss }}\right)<0.2$. In addition, to ensure that the momentum of the reconstructed $Z$ boson and Higgs boson candidates are balanced, the azimuthal separation of the dilepton system and the $E_{T}^{\text {miss }}$ is required to be $\Delta \phi_{Z, E_{T}^{\text {miss }}}>2.6$ and the fractional $p_{T}$ difference should satisfy $\left|E_{T}^{\text {miss }}-p_{T}^{l l}\right| / p_{T}^{l l}<0.2$. Finally events with reconstructed jets are vetoed.

The backgrounds from $Z \rightarrow l l, W$ and multijet production are estimated using data-driven methods. The $Z \rightarrow l l$ background is estimated using side-band regions defined by reversing either the $\Delta \phi\left(E_{T}^{\text {miss }}, p_{T}^{\text {miss }}\right)$, the $\left|E_{T}^{\text {miss }}-p_{T}^{l l}\right| / p_{T}^{l l}$ cut or both cuts. The $W$ and multijet backgrounds are estimated by using a data-driven method based on a control regions containing combinations of real and fake leptons. Efficiencies for identifying a real or fake lepton using the tighter identification are then applied to these control regions to estimate contribution from this background in the signal region. Additionally, the $W W, t \bar{t}, W t$ and $Z \rightarrow \tau \tau$ backgrounds are estimated using the lepton flavour symmetry of the final states - this allows signal free $e \mu$ control regions to be defined in data and the MC normalisation in these control regions to be extrapolated to the $e e$ and $\mu \mu$ signal regions. Finally the $Z Z$ and $W W$ backgrounds are estimated using MC.

The selected data and expected events are split into those from the 2011 and 2012 running periods. No significant excess of events is seen over the SM expectation, hence limits are set for two scenarios with invisibly decaying Higgs bosons. Figure 5 shows the $68 \%$ and 95\% CL for the recently observed SM Higgs boson at $m_{H}=125 \mathrm{GeV}$ decaying invisibly, as a function of the branching ratio $B(H \rightarrow$ invisible $)$. Assuming the $\mathrm{SM} Z H$ production rate for a Higgs boson with mass
$m_{H}=125 \mathrm{GeV}$, branching fractions greater than $65 \%$ can be excluded at $95 \%$ CL. The exclusion limit from this direct search agrees well with the upper limit of $B(H \rightarrow$ invisible $)<0.6$ observed within the combined coupling measurements of the SM Higgs boson search channels made by ATLAS [3]. Further upper limits are also set on the cross section times invisible branching ratio of a single invisibly decaying Higgs boson in the mass range $115<m_{H}<300 \mathrm{GeV}$.

\section{Summary}

The latest results for ATLAS searches for beyond the SM Higgs bosons are presented. Direct searches for Higgs bosons within Two Higgs Doublet Models, for neutral and charged Higgs Bosons within the Minimal Supersymmetric SM and for Higgs decays to invisible particles show no significant excesses over the expectations from the SM. Hence ever more stringent limits are set on the parameters of such models.

\section{References}

[1] ATLAS Collaboration, Physics Letters B 716, 1 (2012)

[2] CMS Collatoration, Physics Letters B 716, 30 (2012)

[3] ATLAS Collaboration, ATLAS-CONF-2013-034 http://cds.cern.ch/record/1528170 (2013)

[4] G.B. et al., Phys. Rept. 516, 1 (2012)

[5] N.P. Nilles, Phys. Rep. 110, 1 (1984)

[6] H.E. Haber, G.L. Kane, Phys. Rep. 117, 75 (1985)

[7] S. Heinemeyer, O. Stål and G. Weiglein, Phys.Lett. B710, 201 (2012), 1112.3026

[8] A. Arbey, M. Battaglia, A. Djouadi and F. Mahmoudi, JHEP 1209, 107 (2012), 1207. 1348

[9] ATLAS Collaboration, ATLAS-CONF-2013-027 http://cds.cern.ch/record/1525887 (2013)

[10] ATLAS Collaboration, ATLAS-CONF-2012-158 http://cds.cern.ch/record/1493601 (2012)

[11] ATLAS Collaboration, JHEP 1302, 095 (2013)

[12] ATLAS Collaboration, JHEP 1303, 076 (2013)

[13] ATLAS Collaboration, JHEP 1206, 039 (2012)

[14] ATLAS Collaboration, Eur.Phys.J. C73, 2465 (2013)

[15] ATLAS Collaboration, ATLAS-CONF-2013-011 http://cds.cern.ch/record/1523696 (2013) 